\title{
Intra-pleural tissue plasminogen activator and deoxyribonuclease: An alternative treatment option for pleural infections in specific populations
}

\author{
Rita Medrano MD, Audra Schwalk MD, Ebtesam Islam MD, PhD
}

\begin{abstract}
Pleural infections can be associated with significant morbidity and mortality. Systemic antibiotics and drainage are the standard treatment, but some patients require surgical intervention for complete resolution. For unsuitable surgical candidates, the intra-pleural administration of tissue plasminogen activator and deoxyribonuclease (tPA/DNase) is a potential treatment option for loculated effusions. We present a case of loculated empyema successfully treated with a 3-day course of once daily intra-pleural administration of TPA/ $D N a s e$. Considering the frailty of our patient, we choose the once daily tPA/DNase due to concerns about potential complications, such as increased risk of bleeding. The published trial evidence and administration regimens of intra-pleural tPA/DNase are also discussed.
\end{abstract}

Keywords: tPA/DNAse, loculated pleural effusion, empyema

\section{INTRODUCTION}

Pleural infections affect more than 65,000 patients each year in both the United States and the United Kingdom, and the incidence is increasing. ${ }^{1}$ The mortality rate from pleural infections is between $10 \%$ and $20 \%$; therefore, prompt diagnosis and management are crucial. ${ }^{2}$ The standard treatment of a pleural infection is the administration of systemic antibiotics and drainage with an intra-pleural catheter. This conservative management fails in approximately one third of patients, who then require surgical drainage. ${ }^{1}$ Early surgical intervention is essential and usually very successful. Surgery is a vital treatment option for many pleural infections, such as loculated effusions, but sometimes it cannot be completed, despite being necessary. Some recent studies have shown that a combination of intra-pleural deoxyribonuclease (DNase)

Corresponding author: Ebtesam Islam

Contact Information: Ebtesam.islam@ttuhsc.edu DOI: $10.12746 /$ swrccc.v6i25.482 and intra-pleural tissue plasminogen activator (tPA) improves radiologic imaging and may be associated with reduced hospital length of stay, improved infection control, and a decreased need for surgery. ${ }^{1}$ Here we present a case of an empyema successfully treated with intra-pleural tPA/DNase in addition to systemic antibiotics.

\section{CASE REPORT}

An 80-year-old woman with mental retardation, spastic quadriplegia, and oropharyngeal dysphagia presented with lower abdominal pain for one day. The pain was described as non-radiating, intermittent, and worse with urination. The patient's caretaker also noted she had dark, foul smelling urine and a recent fever. On admission, vital signs were BP 93/56 mmHg, HR 93 beats per minute, RR 20 breaths per minute, temperature $100.5^{\circ} \mathrm{F}$, and $\mathrm{SpO}_{2} 96 \%$ on room air. Physical examination demonstrated suprapubic tenderness. The thoracic examination demonstrated diminished breath sounds on the right when compared to the left, and crackles in the right upper lung region. Admission labs were unremarkable except for WBC $25.1 \mathrm{~K} / \mathrm{UL}$ 


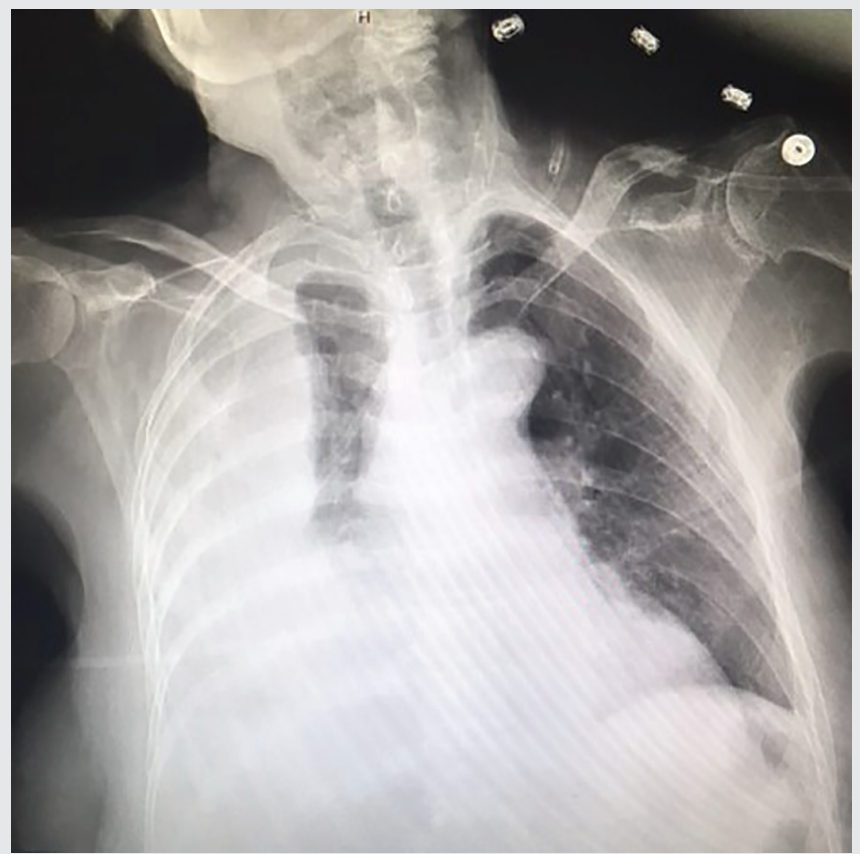

Figure 1. Portable semi-upright chest $\mathrm{x}$-ray showing a large right-sided, loculated pleural effusion with compressive atelectasis of the right lung.

with $73 \%$ neutrophils. Urinalysis was consistent with a urinary tract infection. Computed tomography of the abdomen and pelvis revealed bibasilar atelectasis, a right lower lobe infiltrate and a right-sided pleural effusion, but no evidence of pyelonephritis. Upon further questioning, the patient had had a non-productive cough for about one week prior to presentation. She was admitted to the hospital and started on empiric IV antibiotics to cover a urinary tract infection and suspected aspiration pneumonia. Her chest x-ray progressively worsened over the next three days as she developed a large, right-sided loculated pleural effusion with compressive atelectasis (Figure 1). Therapeutic thoracentesis was then attempted but yielded only $100 \mathrm{cc}$ of cloudy, yellow fluid, due to increased viscosity preventing adequate flow. The pleural fluid analysis was compatible with an empyema (Table).

The patient's medical decision maker opted against surgical treatment; therefore, alternative options were provided. The decision was made to administer tPA/ DNase through a pigtail catheter inserted in the chest
Table. Pleural fluid analysis

\begin{tabular}{|l|l|}
\hline Color & Yellow \\
\hline Clarity & Cloudy \\
\hline RBC & $3687 / \mathrm{mm}^{3}$ \\
\hline WBC & $14680 / \mathrm{mm}^{3}$ \\
\hline Neutrophils & $94 / \mathrm{mm}^{3}$ \\
\hline Lymphocytes & $6 / \mathrm{mm}^{3}$ \\
\hline Albumin & $1.9 \mathrm{~g} / \mathrm{dl}$ \\
\hline Glucose & $7 \mathrm{mg} / \mathrm{dl}$ \\
\hline LD & $1831 \mathrm{IU} / \mathrm{L}$ \\
\hline
\end{tabular}

cavity. The dose of tPA was $10 \mathrm{mg}$; the dose of DNase was $5 \mathrm{mg}$. These medications were given once daily, followed by clamping of the chest tube for one hour after each injection. After one dose of the medications, the chest tube drained $1300 \mathrm{~mL}$ of yellow fluid, and the patient's shortness of breath and chest $x$-ray significantly improved. On day 2 the chest tube drained $550 \mathrm{~mL}$ of fluid, and on day 3 the chest tube drained less than $100 \mathrm{~mL}$ of fluid. The chest tube was removed after day 3 with significant improvement in her chest $\mathrm{x}$-ray and clinical condition (Figure 2). The patient was discharged home with routine follow-up and no complications to date.

\section{Discussion}

Pleural infections are a frequently encountered clinical problem with potentially high morbidity and mortality. The standard treatment for pleural infection includes prompt drainage and appropriate antibiotic therapy. ${ }^{3}$ Surgical decortication with thoracotomy or VATS is often necessary for the treatment of more complicated pleural infections, like empyema, or when more conservative therapy is unsuccessful. In general, exudative pleural effusions have decreased fibrinolytic activity, high TNF alpha and plasminogen activator inhibitor concentrations, and a decreased level of tPA. ${ }^{4}$ Alternative options for the treatment of infected pleural effusions have been investigated for use when surgical debridement cannot be pursued. The most promising treatment is the use of intra-pleural tPA/ DNase. The goal when using this fibrinolytic therapy is 


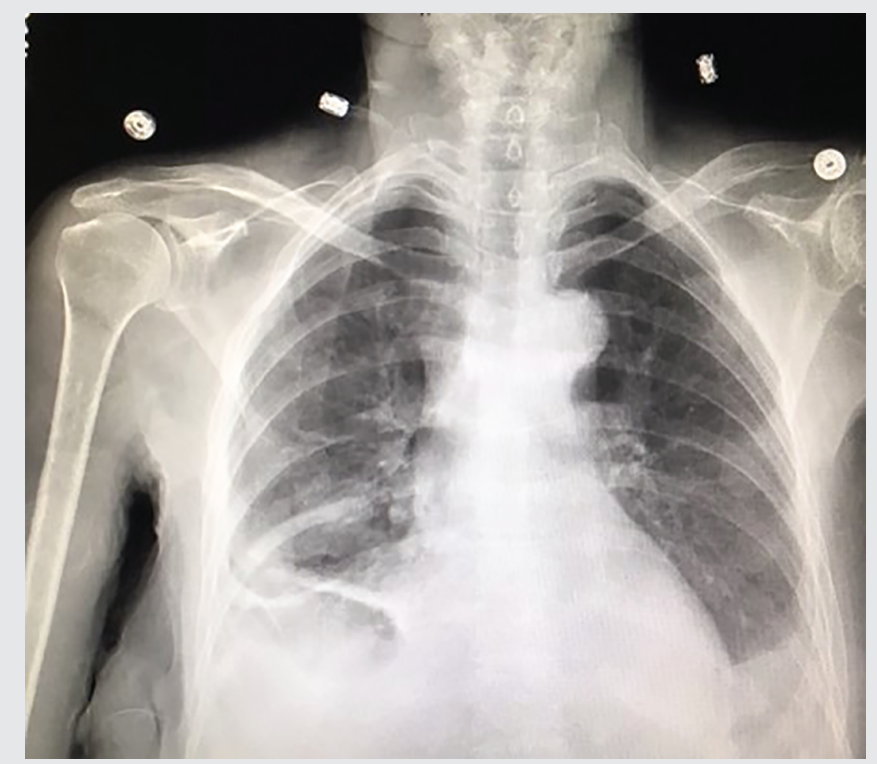

Figure 2. Portable chest x-ray taken on day 3 of treatment showing significant improvement in the previously seen right-sided pleural effusion with expansion of the right lung.

to lyse loculations and fibrin deposits to decrease the viscosity of the fluid and facilitate adequate drainage.

The MIST 1 trial was the first large, multi-center study to evaluate the administration of intra-pleural fibrinolytic agents for the drainage of infected pleural fluid. This double-blinded study included 454 patients and evaluated the potential benefit of intra-pleural streptokinase versus placebo, but the results were not promising. Intra-pleural streptokinase was ineffective in reducing mortality, the need for surgical drainage, and decreasing the hospital length of stay. ${ }^{3}$

The MIST 2 trial with 210 patients compared the results of four study groups: double placebo, intra-pleural tPA and placebo, DNase and placebo, or the combination of intra-pleural tPA/DNase. The primary outcome was the change in pleural opacity, but surgical rates, hospital length of stay, and adverse events were also investigated. This study found that the combination of intra-pleural tPA/DNase significantly improved fluid drainage when compared to the other treatments and was associated with a reduced need for surgery and a decreased hospital length of stay. The doses of DNase and tPA used were $5 \mathrm{mg}$ and $10 \mathrm{mg}$, respectively. These medications were each administered twice daily for three days, and after each intra-pleural injection, the chest tubes were clamped to allow these medications to dwell in the pleural space for one hour. ${ }^{1}$

The administration protocol used in the MIST 2 trial differs from the one we frequently use at our institution. We successfully used a 3-day regimen of once daily intra-pleural tPA/DNase to treat complicated infected pleural effusions and empyema. Our patient received daily doses of these intra-pleural medications with excellent radiographic and clinical improvement. A recent study of 55 patients from the University of Florida also showed that once daily intra-pleural tPA/DNase for three consecutive days can be safe and effective in patients with empyema or complicated parapneumonic effusions. ${ }^{5}$

\section{Conclusion}

The administration of intra-pleural tPA and DNase is a potential treatment option for complicated parapneumonic effusions and empyema in patients for whom standard medical management has failed and thoracic surgery is not a feasible alternative. The abbreviated dosing regimen of once daily administration may prove to be just as successful as twice daily dosing and potentially decreases side effects of these medications, such as bleeding, and the time required for twice daily dosing. This case illustrates the utility and feasibility of tPA/DNase for the treatment complicated pleural effusions in patients with high or prohibitive surgical risks. More studies are needed to establish the optimal intra-pleural dosing regimen and patient selection.

\section{Key Statements}

1. This study demonstrates the efficacy of once a day dosing of tPA/DNAse.

2. Future studies should include once a day tPA/ DNAse treatment arms and consider the possibility of decreasing the dose of tPA. 
Article citation: Medrano R, Schwalk A, Islam E. Intra-pleural tissue plasminogen activator and deoxyribonuclease: An alternative treatment option for pleural infections in specific populations. Southwest Respiratory and Critical Care Chronicles 2018;6(25):38-41

From: Department of Internal Medicine, Texas Tech University Health Sciences Center, Lubbock, TX

Submitted: $12 / 1 / 2017$

Accepted: 4/8/2018

Reviewer: Victor Test MD

Conflicts of interest: none

This work is licensed under a Creative Commons Attribution-ShareAlike 4.0 International License

\section{REFERENCES}

1. Rahman NM, Maskell NA, West A, et al. Intra-pleural use of tissue plasminogen activator and DNase in pleural infection. N Engl J Med 2011;365:518-526.

2. Rahman NM, Kahan BC, Miller RF, et al. A clinical score (RAPID) to identify those at risk for poor outcome at presentation in patients with pleural infection. Chest 2014;145: 848-855.

3. Maskell NA, Davies CW, Nunn AJ, et al. for the First Multicenter Intra-Pleural Sepsis Trial (MIST1) Group. U.K. Controlled trial of intra-pleural streptokinase for pleural infection. N Engl J Med 2005;352:865-874.

4. Piccolo F, Popowicz N, Wong D, et al. Intrapleural tissue plasminogen activator and deoxyribonuclease therapy for pleural infection. J Thoracic Disease 2015;7(6):999-1008.

5. Mehta H J, Biswas A, Penley A M, et al. Management of intra-pleural sepsis with once daily use of tissue plasminogen activator and deoxyribonuclease. Respiration 2016; 91:101-106. 